\title{
IT Services $=$ People + Tools + Processes
}

\author{
People add the power to a simple equation \\ for enlightened decision-making in the IT service industry
}

By Kemal A. Delic

The evolution of the world's economy toward an economy of services is a well-known, century-long social phenomenon. Some authors go so far as to associate the level of wealth and well being-ness of a nation with the percentage of the workforce employed in the service industries. The information technology (IT) segment of the economy of services has exhibited substantial growth over the past few decades. The IT industry has typically grown two to three times faster than GDP. Even now, in difficult and uncertain economic circumstances, IT remains a growth business.

A frequent definition of IT services is "IT Services = People + Tools + Processes". It is an abstraction that implies that IT services are the typical outcome of people's activities using IT tools according to the precisely defined processes. This simple yet powerful abstraction might give us valuable insight into possible approaches to the management of IT services. It should be treated as a tricky combination of the science of large-scale, interactive social systems involving well engineered, dependable tools with practical management aims of defining business practice as the set of hierarchical, embedded business processes.

People are the most relevant part, bringing their intelligence into the service equation. They are also the most expensive part but the very key for overall success. When treated separately from tools and processes, people's group behavior can be better understood. But once they become intertwined with tools and processes, people's behaviors become unpredictable, changed and biased. They are exposed to training, skill improvements, learning from mistakes and being the attraction point of each and every knowledge management activity.

Tools offer augmentation power, certain levels of automation, and attractive, obvious benefits. They also exhibit (sometimes) a vendor's locking power and (hidden) high total cost of ownership. Tools typically solve problems in a narrow limited domain and do not 
account for the holistic aspect of the overall enterprise efficiency and efficacy. Choice of the tools is crucial for the majority of IT technology-based savings.

Processes are usually products of the corporate, business and/or group policies and reuse of successful experiences (sometimes considered as elementary knowledge management). They are an important but quite often inefficient and underutilized part of the IT equation. This could be partially explained by the fact that they are created and reused by (unpredictable) people. The new paradigm of enterprise Web services attempts to address the automatic capturing and reuse of business processes.

This simplistic equation might be a useful conceptual abstraction mechanism for managers, technologists, architects and researchers. Managers may use it for cost management purposes within the TCO (total cost of ownership) context. Technologist might use it for ROI (return on investments) arguments proposing investment in new IT tools. Capacity management is a typical concern of the enterprise architects dealing with data, network and operation centers. Static and dynamic aspects of this complex domain could be modeled after this equation. Finally, researchers interested in modeling of IT services might use it as an originating point for complex supply chain systems, factored into three components.

On yet another axis, reading of this equation and its usage in different circumstances and environments could be quite different depending, for example, upon who is delivering IT services:

1. Internal corporate IT (typically, considered as the corporate cost/overhead function) or

2. IT outsourcing business (characteristically, offering predictable pricing and service quality)

The same equation will be seen and judged differently by the same professionals working in different business contexts and specifically biased mind-sets. Depending upon who uses it and for what purpose, each equation component will have distinct meaning, varying evolution dynamics and different cost drivers.

To better illustrate this argument, let's abstract the corporate IT department as a functional block containing a corporate help desk, an operational center and a data center interconnected with corporate network (Figure 1.). 


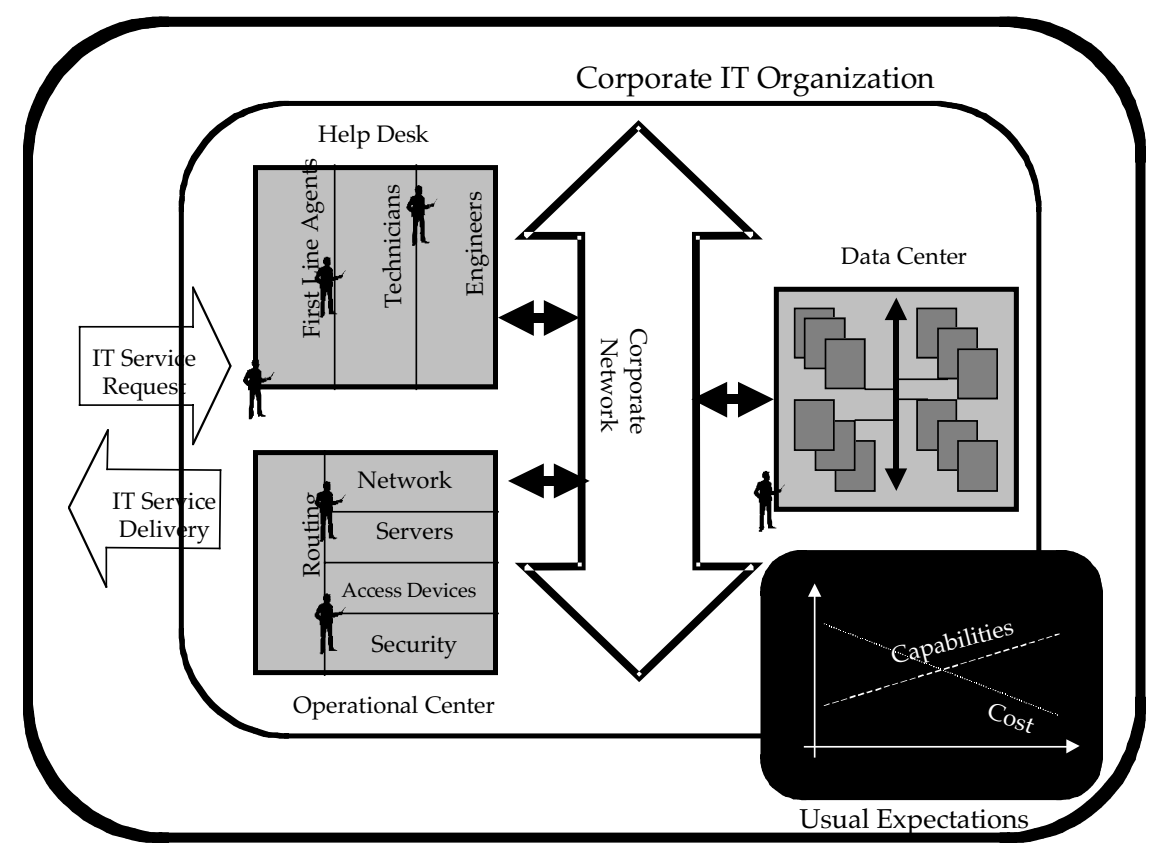

Figure 1. Corporate IT Services

Corporate services will be requested from and delivered by the corporate IT organization. Expectations are that IT capabilities will constantly grow while the cost will be gradually reduced. As the major cost factor is staff salary, each corporate sub-domain will have performance ratios such as calls/analyst, applications/head and servers/head (as the most frequent decision-making factor). Considering that IT organization can be internal or partially/completely outsourced, the logic of the decision-making will be very different. The size of investments into enterprise tools, for example, will be very different for single-customer tools versus multiple-customers tools. Economy of scale will give benefits but additional costs will be created by increased concerns for security and separation of customers. Investments into analysts' training will have different aspects for an internal IT person versus a person hired by an outsourcer. Consequently, internal and outsourced IT organizations function on different economic and technology logic.

The number of influencing factors in both cases is huge, the domain of applications is complex, and in-depth understanding of real-life IT problems is extremely challenging. Therefore, it is useful to split the IT service equation into three pieces and apply it in two different situations. It is useful not only for understanding of simple services such as corporate e-mail but also for complex services such as corporate help-desk or data center. Recent developments in Web service, utility computing and adaptive enterprise services 
provide yet another perspective in which the given IT equation might have an intriguing application.

\section{Conclusion}

This simple, yet powerful equation might explain the hard choices to be made in the estimated $\$ 400$ - 600 billion per year IT services market. It is analogous to the formula "Programs = Algorithms + Data Structures", which is often used in the fields of information theory and software engineering. However, programs do not explicitly include people in the equation. In the IT service equation, however, people are the principal cost factor, the unique source of intelligence, and the strongest factor of uncertainty. The IT industry service players that understand and properly apply this simple yet powerful service equation within their specific business context will be the future winners and gainers in the always-growing IT services market.

Kemal Delic(kemal_delic@hp.com) is a lab scientistwith Hewlett-Packard's R\&D operations and a senior enterprise architect with experience in knowledge management, Bayesian nets modeling, and realtime intelligent systems.

Source: Ubiquity, Volume 4, Issue 37, Nov. 12 - 18, 2003, http://www.acm.org/ubiquity/ 